Document downloaded from:

http://hdl.handle.net/10251/158854

This paper must be cited as:

Giménez-Marqués, M.; Santiago-Portillo, A.; Navalón Oltra, S.; Alvaro Rodríguez, MM.; Briois, V.; Nouar, F.; García Gómez, H.... (2019). Exploring the catalytic performance of a series of bimetallic MIL-100(Fe, Ni) MOFs. Journal of Materials Chemistry A. 7(35):2028520292. https://doi.org/10.1039/c9ta01948k

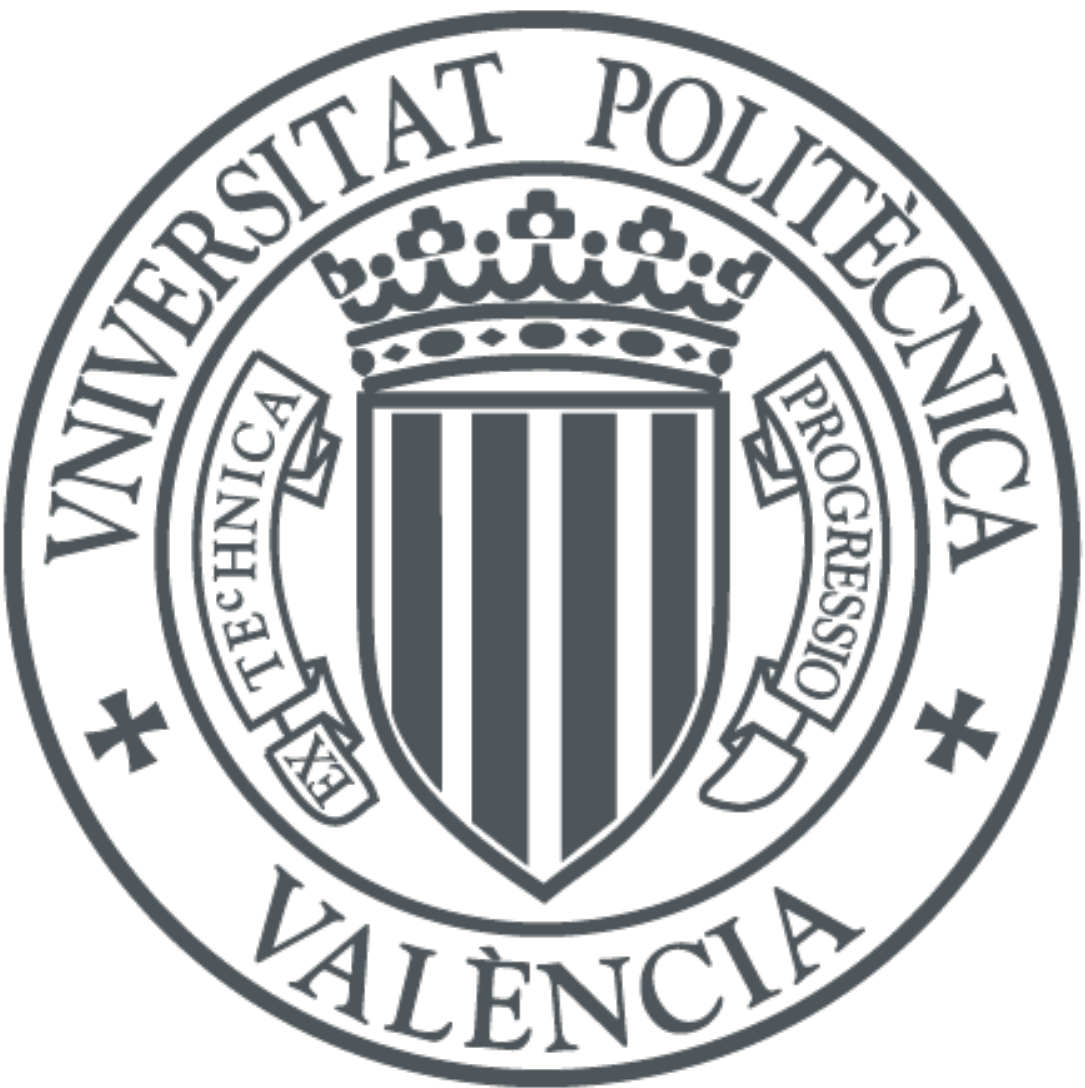

The final publication is available at https://doi.org/10.1039/c9ta01948k

Copyright The Royal Society of Chemistry

Additional Information 


\section{Exploring the catalytic performance of a series of bimetallic MIL- 100(Fe, Ni) MOFs}

Received 00th January 20xx, Accepted 00th January 20xx DOI: $10.1039 / x 0 x \times 00000 x$
Mónica Giménez-Marqués, *a,b Andrea Santiago-Portillo, ${ }^{c}$ Sergio Navalón, ${ }^{c}$ Mercedes Álvaro, ${ }^{c}$ Valérie Briois, ${ }^{d}$ Farid Nouar, ${ }^{a}$ Hermenegildo Garcia, ${ }^{* c}$ Christian Serre. ${ }^{* a}$

A series of mixed-metal Fe"I/Ni" MOFs of the MIL-100 type metal-organic framework (MOF) containing different metal ratios have been synthesized de novo, following an approach that requires tuning of the $\mathrm{Fe}^{\prime \prime \prime} / \mathrm{Ni}^{\prime \prime}$ reactivity. The resulting heterometallic MIL-100(Fe, Ni) materials maintain thermal, chemical and structural stability with respect to the parent MIL100 (Fe) MOF as can be deduced from various techniques. The nature and the oxidation state of the accessible metal cations have been evaluated by in situ infrared spectroscopy and extended X-ray absorption fine structure measurements. The obtained mixed-metal MOFs and the parent material have been evaluated as heterogeneous catalysts in a model acidcatalyzed reaction such as the Prins reaction. It is found that the catalytic activity improves more than one order of magnitude upon incorporation of $\mathrm{Ni}^{\prime \prime}$, with a complete selectivity for the formation of nopol. This increase in the catalytic activity upon incorporation of $\mathrm{Ni}^{\prime \prime}$ correlates with the enhancement in the Lewis acidity of the material as determined by $\mathrm{CO}$ adsorption. The heterometallic MOF can be recycled without observation of metal leaching, while maintaining the crystal structure under the reaction conditions.

\section{INTRODUCTION}

The development of multifunctional solid catalysts is currently of foremost relevance in the field of heterogeneous catalysis. The reason is that it is expected that bi/multi-functionalization can be used to promote cascade or tandem reactions in one single step, thus noticeably decreasing processing costs. In this context, metal-organic frameworks (MOFs) can be leading materials, given their chemically modulable nature and structural diversity, and their already proved suitable performance as solid heterogeneous catalysts for specific liquid phase chemical transformations. 1, 2, 3, 4, 5 Multifunctional MOFs presenting different ligands and/or cations have been already used $n$ catalysis $6,7,8,9$, some of these materials being able to promote one-pot tandem reactions. ${ }^{10,11,12,13,14}$

The real challenge in the synthesis of stable mixed-metal MOFs consists in the control of the metal stoichiometry particularly for cations with different oxidation states and therefore distinct chemical reactivities..$^{15,16}$ In addition, most of the new multimetallic MOFs present unsatisfactory chemical stability under catalytic conditions, an issue that has to be addressed before MOF catalysts can meet industrial applicability. Direct post-synthetic methods (PSM) provide convenient strategy for the preparation of mixedmetal MOFs that has been widely explored recently. ${ }^{17,} 18$ This PSM approach has been successfully applied to partially replace in MOFs

\footnotetext{
a. Institut des Matériaux Poreux de Paris, UMR 8004 CNRS, École Normale Supérieure, École Supérieure de Physique et de Chimie Industrielles de la ville de Paris, PSL University, 75005 Paris, France.

b. Instituto de Ciencia Molecular, Universidad de Valencia, Catedrático José Beltrán 2, 46980 Paterna, Spain.

c. Departamento de química e Instituto de Tecnología Química (ITQ-CSIC-Universitat Politècnica de València, C/Camino de Vera, $s / n, 46022$, Valencia, Spain).

d. Synchrotron SOLEIL-UR1, 91192, Gif-Sur-Yvette, France

Electronic Supplementary Information (ESI) available: [details of any supplementary information available should be included here]. See DOI: 10.1039/x0xx00000x
}

some of the cations by ion metathesis, or to directly coordinate metal complexes in the framework. ${ }^{19,20,21}$

A more recently developed strategy consists in the direct synthesis of bi- or multimetallic MOFs using a straightforward method with predesigned mixed-metal subunits as starting materials. This approach has been denoted as the secondary building unit (SBU) approach. ${ }^{22}$ These SBU methods have been successfully used in the synthesis of porous pure cationic iron(III) dicarboxylate (MIL-88s) ${ }^{23}$ (MIL stands for Materials of Institut Lavoisier) or zirconium(IV) dicarboxylate MOFs (UiO-66's) ${ }^{24}$ (UiO stands for Universitetet i Oslo) and have been recently applied for the preparation of mesoporous mixed-metal MIL-127 MOF or soc-MOF, 25 as well as for the preparation of different iron-containing robust MOFs with a preserved $\mathrm{Fe}^{\prime \prime \prime \prime}{ }_{2} \mathrm{M}^{\prime \prime} \mathrm{O}$ metal cluster core $\left(\mathrm{M}^{\prime \prime}=\mathrm{Co}^{2+}, \mathrm{Ni}^{2+}, \mathrm{Mg}^{2+}\right) \cdot{ }^{26,27}$ These later examples considered the use of mixed-metal acetate building blocks, i.e. the trimeric oxocentered bimetallic $\mathrm{Fe}^{\mathrm{II \prime}} / \mathrm{M}^{\prime \prime}$ acetate system $\left[\mathrm{Fe}_{2} \mathrm{M}\left(\mu_{3}-\mathrm{O}\right)\left(\mathrm{CH}_{3} \mathrm{COO}\right)_{6}\right]\left(\mathrm{M}=\mathrm{Co}^{2+}, \mathrm{Ni}^{2+}, \mathrm{Mn}^{2+}, \mathrm{Zn}^{2+} \ldots\right)^{28}$ obtained from the pure iron(III) oxo acetate cluster. ${ }^{29}$ In this context, we have devoted particular efforts to the preparation of the archetypal mesoporous mixed-metal MIL-100( $\left.\mathrm{Fe}_{2} \mathrm{M}\right)$ material using the predefined mixed-metal acetate building blocks. The main reason to select MIL-100( $\left.\mathrm{Fe}_{2} \mathrm{M}\right)$ is the ability of its parent MIL-100(Fe) solid to perform as heterogeneous catalyst in a large variety of reactions, leading in many cases to large conversions and selectivity. The advantage in obtaining this particular MOF derive from its valuable features: i) MIL-100(Fe) exhibits a very high surface area and pore volume allowing easy diffusion of substrates and products of molecular dimensions smaller than the pore windows; ii) it can be synthesized in large-scale following green methodologies and at low temperature; iii) its robust porous structure possesses a suitable chemical and thermal stability. Following the rational mixed-metal building block approach, we could obtain MIL-100( $\left.\mathrm{Fe}_{2} \mathrm{Ni}\right)$ with an 
appropriate crystallinity with however several drawbacks: i) the formation of undesired iron oxides during the synthesis, which considerably affect the properties of the material with regard to its use as catalyst; ii) a dramatic reduction of chemical stability with respect to the parent robust MIL-100(Fe). These inconveniences prevented the obtained MIL-100( $\left.\mathrm{Fe}_{2} \mathrm{Ni}\right)$ material from any practical application and prompted us to explore alternative synthetic routes leading to the incorporation of low Ni content in heterometallic MIL$100(\mathrm{Fe}, \mathrm{Ni})$ materials. So far, to our knowledge, only mixed metal(III) MIL-100 (Sc combined with Al, Fe, Cr) materials ${ }^{12}$ and MIL-100(Fe, $\mathrm{Mn})^{30}$ have been obtained.

Herein we present the direct synthesis of a series of mixed-metal MIL-100(Fell', Ni") MOFs containing different proportions of $\mathrm{Ni}^{\prime \prime}$. The method follows a synthetic route previously developed by some of $\mathrm{us}^{31}$ in which the preparation of a mixed-metal MIL-53(Cr"I, Fe' $\left.{ }^{\prime \prime \prime}\right)$ required to adjust the metal reactivity to permit the more inert $\mathrm{Cr}^{\text {III }}$ cation to enter into the framework. The mixed-metal MIL-100(Fe, Ni) MOFs obtained here is compared with their parent MIL-100(Fe) material as heterogeneous catalysts in the Prins reaction between $B$ pinene and paraformaldehyde. ${ }^{32}$

\section{RESULTS AND DISCUSSION}

Synthesis and characterization of mixed-metal MIL-100(Fe, Ni) MOFs. The synthesis of a series of heterometallic MIL-100(Fe'II, $\mathrm{Ni")} \mathrm{(1-3)} \mathrm{MOFs} \mathrm{with} \mathrm{three} \mathrm{different} \mathrm{Fe/Ni} \mathrm{ratios} \mathrm{was} \mathrm{carried} \mathrm{out}$ by direct mixing $\mathrm{Fe}^{0}$ with trimesic acid in the presence of aqueous solutions of $\mathrm{HNO}_{3}$ and $\mathrm{Nill}$ salt. Similarly, pure MIL100 (Fe'II) was obtained using $\mathrm{Fe}^{0}$ as the only metal precursor. All materials were obtained in stoichiometric metal-to-ligand amounts, using water as the solvent and stirring for $24 \mathrm{~h}$ at relatively low temperatures $\left(80^{\circ} \mathrm{C}\right)$, avoiding in this case the addition of HF. ${ }^{33}$ Under these conditions, the Fe $\mathrm{Fe}^{\text {III }}$ cations are very slowly released in the reaction media, permitting to some extent the incorporation into the framework of the less favoured acidic $\mathrm{Nil}$ cation that is $100 \%$ available since the early stage of the reaction, in large $\mathrm{Ni}$ to Fe excess. Three different $\mathrm{Fe}$ "II/Ni" metal ratios were considered, obtaining the bimetallic MIL-100(Fe, Ni) (1-3) MOF materials whose analytical data are summarized in Table 1.

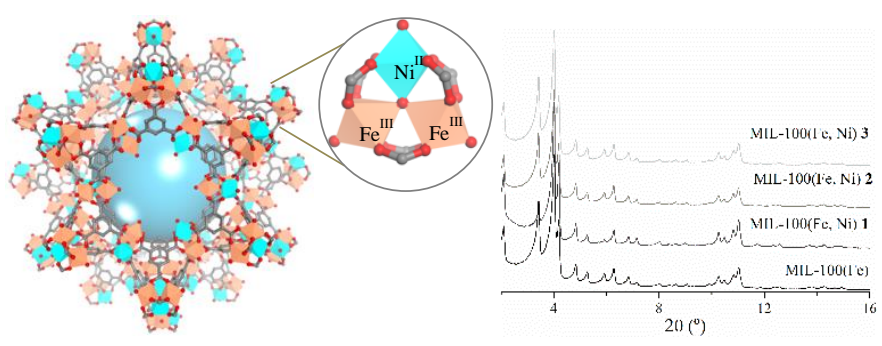

Figure 1. (Left) Structure of the heterometallic MIL-100(Fe, Ni) and trimeric heterometallic unit. Iron and nickel octahedra, carbon and oxygen atoms are in orange, blue, grey and red, respectively. (Right) Powder X-ray diffraction patterns of mixed-metal MIL-100(Fe, Ni) 13 materials as compared with bare MIL-100(Fe) material.
Table 1. Summary of the Ni content, specific surface area and metal composition of the obtained heterometallic MOFs.

\begin{tabular}{|c|c|c|c|c|c|}
\hline \multirow{2}{*}{\multicolumn{3}{|c|}{$\% \mathrm{Ni}^{\prime \prime} *$}} & \multirow{2}{*}{$\underset{\left(\mathrm{m}^{2} \cdot \mathrm{g}^{-1}\right)}{\text { aвET }}$} & \multicolumn{2}{|c|}{$\%$ TGA based } \\
\hline & & & & inorganic & organic \\
\hline MIL-100(Fe) & theoretical & & - & 37.2 & 62.8 \\
\hline $\mathrm{Fe}_{3} \mathrm{O}\left(\mathrm{NO}_{3}\right)\left[\left(\mathrm{CO}_{2}\right)_{3}\left(\mathrm{C}_{6} \mathrm{H}_{3}\right)\right]_{2}$ & experimental & & 1600 & 36.7 & 63.3 \\
\hline M.W. $659.77 \mathrm{~g} \cdot \mathrm{mol}^{-1}$ & & & & & \\
\hline $\begin{array}{c}\left.\text { MIL-100( } \mathrm{Fe}_{2} \mathbf{N i}\right) \\
\mathrm{Fe}_{2} \mathrm{NiO}\left[\left(\mathrm{CO}_{2}\right)_{3}\left(\mathrm{C}_{6} \mathrm{H}_{3}\right)\right]_{2}\end{array}$ & theoretical & & & 30.7 & 69.3 \\
\hline M.W. $597.77 \mathrm{~g} \cdot \mathrm{mol}$ & & & & & \\
\hline 1 & experimental & $1.0 \pm 0.3$ & 1545 & 33.5 & 66.5 \\
\hline 2 & & $3.1 \pm 0.2$ & 1525 & 33.7 & 66.3 \\
\hline 3 & & $5.0 \pm 0.2$ & 1570 & 34.1 & 65.9 \\
\hline
\end{tabular}
performed by considering $100 \%$ weight at $200^{\circ} \mathrm{C}$ corresponding to the dried samples.

Further attempts to obtain mixed-metal MIL-100 included postsynthetic ion metathesis of MIL-100(Fe) by soaking the crystalline material in concentrated aqueous $\mathrm{Ni}$ "l salt solutions, as well as direct mixture of the corresponding inorganic $\mathrm{Fe}^{\mathrm{III}}$ and $\mathrm{Ni}$ "l salts with trimesic acid, were carried out. In all cases, the single metal MIL-100(Fe) material was obtained regardless either the $\mathrm{M}^{\prime \prime}$ concentration or the immersion time. This failure to obtain the mixed-metal MIL-100(Fe, $\mathrm{Ni})$ under these conditions is likely due to the preferential interaction of Fe"ll hard cations with carboxylates respect to soft cations (i.e. Ni"). The obtained heterometallic MIL-100(Fe, Ni) MOFs 1-3 and the corresponding homometallic MIL-100(Fe) were characterized by powder X-ray diffraction (PXRD), Fourier transform infrared spectroscopy (FT-IR), thermogravimetric analyses (TGA), inductively coupled plasma-atomic emission spectroscopy (ICP-AES), $\mathrm{N}_{2}$ sorption measurements, scanning electron microscopy (SEM) and extended X-ray absorption fine structure (EXAFS). As seen by PXRD, all powder diffraction patterns of the as-synthesized MIL-100(Fe) and mixed-metal MIL-100(Fe, Ni) (1-3) samples match that of the simulated MIL-100(Fe) material, confirming the formation of isostructural single-phase MIL-100 material (Figure 1).

Metal content of the mixed-metal MOFs was determined by ICP-OES analyses and revealed increasing amounts of $\mathrm{Ni}$ in the range 1 to $5 \%$, respectively for samples 1-3. These metal compositions differ from the initial metal stoichiometry used in the synthetic conditions (see experimental section), evidencing the difficulty in incorporating a divalent cation into the metal nodes of MIL-100. This fact can be attributed to the structural instability generated in the MIL-100(Fe) framework when the larger divalent $\mathrm{Ni}^{\prime \prime}$ cation is incorporated leading to a neutral trimeric oxo-cluster unit (Figure 1). In an effort to increase the percentage of incorporated divalent $\mathrm{Ni}$, a synthetic route based on the $\mathrm{SBU}$ approach was followed using the preformed mixed-metal $\mathrm{Fe}_{2} \mathrm{Ni}\left(\mu_{3}-\mathrm{O}\right)\left(\mathrm{CH}_{3} \mathrm{COO}\right)_{6}$ oxo-acetate trimer as metal source, as previously reported by some of us. ${ }^{25} \mathrm{MIL}-100$ (Fe'll, Nill) containing $33 \%$ of $\mathrm{Ni}$ was synthesized, leading to the formation of an amorphous gel-like material with a much lower specific surface area $\left(\mathrm{S}_{\mathrm{BET}}=700 \mathrm{~m}^{2} \cdot \mathrm{g}^{-1}\right)$. Similar results have been recently obtained in a series of acetate-based MOFs using in this case the acetate of trimeric $\mathrm{Cr}_{3}\left(\mu_{3}-\mathrm{O}\right)$ building block and biphenyl-4,4-dicarboxylic acid $\left(\mathrm{H}_{2-}\right.$ BPDC). ${ }^{34}$

TGA analysis performed on the solids revealed comparable thermal stability up to $300^{\circ} \mathrm{C}$ with weight losses of the mixed-metal MOFs in agreement with the expected range: about $66 \%$ ligand loss for MIL$100(\mathrm{Fe}, \mathrm{Ni}) 1-3\left(63 \%\right.$ and $69 \%$ calc. for the $\mathrm{Fe}_{3} \mathrm{O}$ and $\mathrm{Fe}_{2} \mathrm{NiO}$ ratio, 
respectively) (see Table 1 and Figures S1-S4). FT-IR analyses on MOFs 1-3 also confirmed the formation of the MIL-100 structure. All materials exhibited the characteristic asymmetric and symmetric stretching bands of the carboxylate anions at $1630-1576 \mathrm{~cm}^{-1}$ and 1450-1382 $\mathrm{cm}^{-1}$, respectively. The metal-oxygen bands of the $\mathrm{M}_{3}\left(\mu_{3}\right.$ O) group were identified at $624 \mathrm{~cm}^{-1}$ in MIL-100(Fe) corresponding to the $\mathrm{Fe}_{3} \mathrm{O}$ trimeric unit, whereas the appearance of a new band at 569 $\mathrm{cm}^{-1}$ in 1-3 attributed to the $\mathrm{Fe}_{2} \mathrm{NiO}$ unit was recorded for the mixed metal samples (see Figure S5). Chemical stability of the obtained MIL$100(\mathrm{Fe}, \mathrm{Ni})$ 1-3 materials was evaluated in water as compared to the bare MIL-100(Fe) material. After $24 \mathrm{~h}$ of stirring at room temperature, none of the materials presented leaching of $\mathrm{Ni}$ or Fe metals $(<1 \%)$ and maintained their crystallinity (Figure S6). Upon water treatment at $80{ }^{\circ} \mathrm{C}$ a leaching of $c a .15-20 \%$ of the total $\mathrm{Ni}$ content was observed for materials 1-3, whereas no Fe leaching was detected. Similar studies conducted in $\mathrm{MeCN}$ at $80{ }^{\circ} \mathrm{C}$ revealed negligible $\mathrm{Ni}$ or Fe metal leaching $(<1 \%)$.

The porosity of the solids was characterised by $\mathrm{N}_{2}$ sorption analysis. Figure 2 shows the isotherms of MIL-100(Fe) and MIL-100(Fe, Ni) 1-3 revealing characteristic type I isotherm expected for the MIL-100 microporous system with preservation of the porosity (see logarithmic scale of pressure range in Figure S3). The obtained accessible surface area values calculated using the BrunauerEmmett-Teller (BET) model are summarized in Table 1. The minor differences encountered in BET areas $\left(1600-1521 \mathrm{~m}^{2} \cdot \mathrm{g}^{-1}\right)$ are in the range of possible experimental error in mass calculation. It is important to mention that a substantial drop of the BET surface area from 1600 to $1180 \mathrm{~m}^{2} \cdot \mathrm{g}^{-1}$ is detected upon further insertion of $\mathrm{Ni}^{\prime \prime}$ cation (10-15\% of metal substitution) (Figures S7 and S8). This decrease in surface area may be related to the presence of occluded metal oxides, which is supported with a larger inorganic percentage detected in thermogravimetric studies.

$\mathrm{CO}$ adsorption experiments were performed to evaluate the effect of the nature and proportion of the different Lewis acid sites present in the framework as compared to the single cationic material.
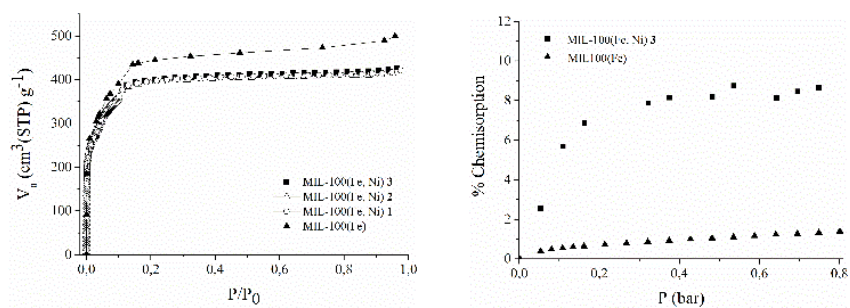

Figure 2. (Left) $\mathrm{N}_{2}$ sorption isotherms for MIL-100(Fe) and heterometallic MIL100(Fe, Ni) 1-3 materials at $77 \mathrm{~K}$. (Right) Chemisorption of CO in MIL-100(Fe) and MIL-100(Fe, Ni) 3 at $303 \mathrm{~K}$. Solids were activated at $423 \mathrm{~K}$ for $12 \mathrm{~h}$ under vacuum. Chemisorption is presented as relative value of the total sorption isotherm.

Heterometallic material $\mathbf{3}$ was selected for these CO adsorption measurements considering its larger $\mathrm{Nill}^{\prime \prime}$ content $(5 \%)$ and preserved porosity. Figure 2 (right) depicts the $\mathrm{CO}$ chemisorption isotherm for sample 3 compared to that of pure MIL-100(Fe) after activation at $423 \mathrm{~K}$ for 12 hours under vacuum. It is important to note that under these conditions, it has been shown previously that Fe metal sites remain in the +3 oxidation state, meaning that the presence of reduced $\mathrm{Fe}^{\mathrm{ll}}$ species is negligible. ${ }^{22,35}$ A noticeably lower $\mathrm{CO}$ chemisorption capacity is observed in this pressure range for the pure iron solid, whereas an increase in the chemisorption capacity of approximately $10 \%$ is measured for the mixed metal MIL-100(Fe, Ni) solid activated under the same conditions. This is consistent with previous studies carried out by some of us on MIL-127(Fe, Ni) materials. ${ }^{25}$ These results point out the effective impact of mixedmetal cations in the Lewis acidity of the material and, therefore, also presumably on its catalytic activity as it will be described below.

SEM and energy dispersive X-ray spectroscopy (EDS) elemental mapping of the heterometallic MIL-100(Fe, Ni) material 3 confirmed the mixed-metal composition revealed by ICP-OES but also endorsed the regular $\mathrm{Fe}$ and $\mathrm{Ni}$ distribution in the material at submicrometric resolution, ruling out the presence of large aggregates of independing $\mathrm{Ni}$ or Fe nanoparticles (Figure S9). This elemental mapping is compatible with the homogeneous spatial distribution at micrometric scale of $\mathrm{Nill}^{\prime \prime}$ cations in the heterometallic MIL-100 material. Further evidence of the structural composition was provided by analysis of the EXAFS spectra of the materials at the ROCK beamline of the SOLEIL synchrotron radiation laboratory. At the Fe $\mathrm{K}$ edge, iron is confirmed at the oxidation state +3 . Least square fittings of EXAFS spectra recorded for sample MIL-100(Fe) (without $\mathrm{Ni}$ ) and heterometallic sample MIL-100(Fe, Ni) 3 (containing $5 \% \mathrm{Ni}$ ) are fully consistent with the MIL-100 reported structure (see Table S1 and Figures S10-17). At the Ni K edge, Ni is confirmed at the oxidation state +2 with the rising edge position at the same energy position than $\mathrm{NiO}$ or $\mathrm{Ni}(\mathrm{OH})_{2}$ references. This conclusion concerning the oxidation state of the metals is similar regardless the Ni loading of the sample.

Least square fitting of EXAFS spectra recorded for materials MIL$100(\mathrm{Fe}, \mathrm{Ni}) \mathbf{2}(3 \% \mathrm{Ni})$ and $\mathbf{3}(5 \% \mathrm{Ni})$ have been performed with the substitution of one iron atom by $\mathrm{Ni}$. It is found very similar structural parameters for second neighbours (carbon) and iron second neighbours than for those characterizing the materials at the Fe Kedge. The first $\mathrm{Ni}-\mathrm{O}$ coordination shell is described with longer distances than $\mathrm{Fe}-\mathrm{O}$, which is consistent with the $\mathrm{Ni}(2+)$ oxidation state. Debye Waller factors are larger for $\mathrm{Ni}$ data than for Fe data likely due to the presence of local distortion upon substitution of Fe $\mathrm{III}^{\mathrm{II}}$ by $\mathrm{Nill}$ in the structure. Therefore, it is established that $\mathrm{Nil}$ atoms are likely replacing Fe"ll positions in the trimeric units of the crystal and they are distributed homogeneously throughout the network.

In situ IR spectroscopy. Analysis of the accessible metal sites. To gain further insights into the nature and the oxidation state of the accessible cations in the framework, in-situ IR analysis was carried out on mixed-metal MOF MIL-100(Fe, Ni) 3, comparing the results with that of pure iron-based MIL-100(Fe) material. Adsorption of NO as molecular probe was monitored by in-situ IR spectroscopy, given the well-known $v(\mathrm{NO})$ bands of adsorbed nitrosyls in the related MIL-127(Fe, Ni) material.25 The infrared spectra were recorded at low temperature $\left(-125^{\circ} \mathrm{C}\right)$ after $\mathrm{NO}$ adsorption on MIL-100(Fe, Ni) and MIL-100(Fe) activated under vacuum at two different temperatures, $150{ }^{\circ} \mathrm{C}$ and $230^{\circ} \mathrm{C}$ (Figure 3). The addition of small NO doses on the activated materials causes the appearance of new absorption bands on the spectra, which are assigned to the adsorbed nitrosyls on coordinatively unsaturated sites (CUS). The results are summarised in Table 2. NO adsorption experiments on the 
heterometallic MIL-100(Fe, Ni) activated at $150{ }^{\circ} \mathrm{C}, \mathrm{MIL}-100(\mathrm{Fe}$, $\mathrm{Ni}) 150^{\circ} \mathrm{C}$, reveal the appearance of two $v(\mathrm{NO})$ bands centred at 1895 and $1868 \mathrm{~cm}^{-1}$. These bands are attributed to NO interacting with $\mathrm{Fe}^{3+}$ and $\mathrm{Ni}^{2+}$ CUS, respectively. ${ }^{25,36}$ The intensity of these bands increases with the amount of NO introduced, this effect being more pronounced in the $\mathrm{Fe}^{3+}$ nitrosyl band (see supplementary material). When the sample is activated at higher temperature (MIL-100(Fe, $\mathrm{Ni}) 230^{\circ} \mathrm{C}$ ), a new band centered at $1805 \mathrm{~cm}^{-1}$ appears, which is assigned to $\mathrm{NO}$ interacting with $\mathrm{Fe}^{2+} \mathrm{CUS}$. These new $\mathrm{Fe}^{2+}$ sites will be generated by thermal reduction of $\mathrm{Fe}^{3+}$ under the activation conditions, as it has been established in related trimeric-based MOF materials. ${ }^{37}$ Similar NO adsorption studies performed on MIL-100(Fe) material activated at $150{ }^{\circ} \mathrm{C}\left(\mathrm{MIL}-100(\mathrm{Fe}) 150^{\circ} \mathrm{C}\right)$ first give rise to a $v(\mathrm{NO})$ band at $1901 \mathrm{~cm}^{-1}$ (Figure 3). Its intensity increases rapidly with the amount of NO introduced whereas its wavenumber shifts to 1895 $\mathrm{cm}^{-1}$ (Figure S18). Then, another very weak band is observed at 1793 $\mathrm{cm}^{-1}$ at the NO equilibrium pressure. As indicated above, these two bands are assigned to $\mathrm{Fe}^{3+}$ and $\mathrm{Fe}^{2+}$ nitrosyl species, respectively. The infrared spectra of MIL-100(Fe) activated at $230{ }^{\circ} \mathrm{C}$ (MIL$100(\mathrm{Fe}) 230^{\circ} \mathrm{C}$ ) and after $\mathrm{NO}$ adsorption, display the expected very intense couple of bands around $1800 \mathrm{~cm}^{-1}$, assigned to NO adsorption on $\mathrm{Fe}^{2+}$ CUS, in agreement with the known formation of supplementary Fell metal sites upon higher activation temperatures. ${ }^{38,39}$ Notably, the $v(\mathrm{NO})$ band at $1868 \mathrm{~cm}^{-1}$ has not been observed in MIL-100(Fe) as it is expected for the pure iron based MOF.

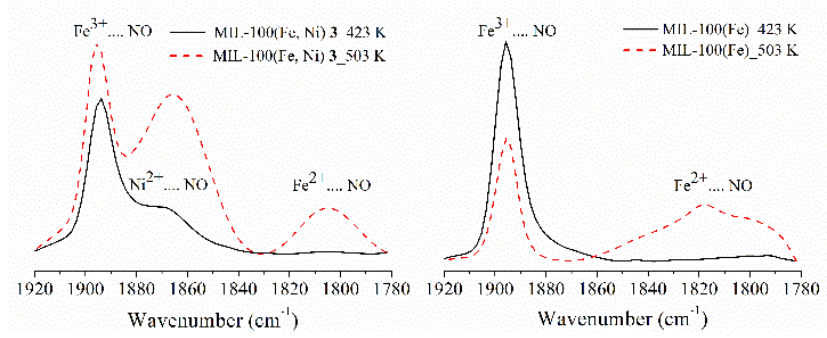

Figure 3. IR spectra of MIL-100(Fe, Ni) 3 (left) and MIL-100(Fe) (right) recorded after introduction of an equilibrium pressure of NO $(300 \mathrm{~Pa})$ at low temperature $(150 \mathrm{~K})$. Spectra after activation of the solid at $423 \mathrm{~K}$ are represented with a black line whereas spectra after activation of the solid at $503 \mathrm{~K}$ are represented in dashed red line. The assignment of the bands has been indicated in the drawings.

Table 2. Summary of the main NO bands attributed to the different M"'-NO and M"-NO nitrosyls compounds formed.

\begin{tabular}{lccc} 
& \multicolumn{3}{c}{$\mathrm{v}(\mathrm{NO}) \mathrm{cm}^{-1}$} \\
& $\mathrm{Fe}^{3+} \ldots \mathrm{NO}$ & $\mathrm{Ni}^{2+} \ldots \mathrm{NO}$ & $\mathrm{Fe}^{2+} \ldots \mathrm{NO}$ \\
\hline MIL-100(Fe, Ni) ${ }_{423 \mathrm{~K}}(3)$ & 1894 & 1868 & -- \\
MIL-100(Fe, Ni) $)_{503 K}(3)$ & 1895 & 1865 & 1805 \\
MIL-100(Fe) $)_{423 K}$ & 1895 & -- & 1794 \\
MIL-100(Fe) & & -- & 1816,1796 \\
\hline
\end{tabular}

This study not only corroborates the efficient incorporation of $\mathrm{Ni}^{2+}$ centers in the heterometallic MIL-100(Fe, Ni) structure based on the observation of free CUS able to interact with NO, but also establishes their accessible Lewis acid character. Importantly, the characteristic partial reduction of $\mathrm{Fe}^{\mathrm{III}}$ in MIL-100 is also evidenced in the heterometallic MIL-100(Fe, Ni) material upon activation at higher temperatures. Generation of $\mathrm{Fe}^{\prime l}$ is likely to be due to the coexistence of both type of trimeric units, pure $\left(\mathrm{Fe}_{3} \mathrm{O}\right)$ and mixed metal $\left(\mathrm{Fe}_{2} \mathrm{NiO}\right)$ trimers, in the structure of MIL-100(Fe, Ni).

Catalytic activity. To determine the distinctive catalytic activity of the obtained heterometallic materials MIL-100(Fe, Ni) 1-3 with respect to that of their parent MIL-100(Fe) material, we tested them as solid catalysts in the acid-catalyzed Prins reaction. ${ }^{40} \mathrm{In}$ particular, the model reaction studied was the synthesis of nopol via Prins condensation of $\beta$-pinene and paraformaldehyde as a model reaction. ${ }^{32}$

Prior to the catalytic reaction, the samples MIL-100(Fe, Ni) 1-3 and MIL-100(Fe) were thermally activated at $150{ }^{\circ} \mathrm{C}$ under vacuum overnight. In a typical procedure, the Prins condensation was carried out with the MIL-100(Fe, Ni) at $80^{\circ} \mathrm{C}$ in $\mathrm{MeCN}$. Catalytic experiments were carried using $0.5 \mathrm{~mol} \%$ (total metal based) of solid (Figure S8). It is worth mentioning that further increase in the amount of catalyst (up to $0.7 \mathrm{~mol} \%$ ) had no significant effect on the reaction rate. Figure 4a shows the temporal evolution of $\beta$-pinene conversion to the corresponding nopol promoted by the mixed-metal MOF and the analogous MIL-100(Fe). After $10 \mathrm{~h}$ of reaction, all materials show a complete selectivity for the formation of nopol, but whereas the use of homometallic MIL-100(Fe) affords < 20\% conversion, the use of heterometallic samples as catalysts afford between 70-100 \% conversion. Indeed, only MIL-100(Fe, $\mathrm{Ni}) 3$ material resulted in a total conversion to nopol after $10 \mathrm{~h}$. Therefore, the activity test clearly shows that the presence of $\mathrm{Ni}$ increases significantly the catalytic activity of MIL-100 as Lewis acid, increasing the initial reaction rate with the percentage of $\mathrm{Ni}$ in the range under study. These catalytic data are in accordance with the increase of the Lewis acidity determined by $\mathrm{CO}$ titration upon incorporation of $\mathrm{Ni}^{2+}$. A plausible explanation for the observed improved catalytic behaviour may be associated with the distortion caused in the framework upon insertion of $\mathrm{Ni}$ "Lewis acid sites, as demonstrated by EXAFS experiments (see Figures S10-17). It is believed that the formation of the mixed-metal trimers in the framework may enable the accessibility of the Fe'll acid Lewis sites, thus enhancing the catalytic activity of the catalyst.

Stability of the best MIL-100(Fe, Ni) 3 catalyst under the reaction conditions was established by recovering and reusing the material in five consecutive runs, observing that the initial reaction rate, final conversion and complete selectivity to nopol were maintained when the material was properly washed (uses 2, 4 and 5) (Figure 4b). X-ray powder diffraction analysis of the fresh and the reused materials (Figure $4 \mathrm{~b}$ inset) confirmed the preservation of the crystallinity upon successive catalytic reactions, whereas analysis of the liquid phase after removal of the solid catalyst established the absence of iron or nickel leaching from the solid to the liquid phase.

The heterogeneity of the catalytic procedure was ascertained by the Sheldon's hot filtration test. Figure 4 shows a comparison of the temporal profile of $\beta$-pinene conversion in the presence of 
MIL-100(Fe, Ni) 3 and upon hot filtration of the solid catalyst after 1 hour reaction. Noticeably, the reaction did not proceed after removal of the catalyst, demonstrating that no catalytically active metal species are present in the filtrate and therefore establishing the heterogeneity of the MOF. A productivity test using a large excess of $\beta$-pinene $(10 \mathrm{mmol})$ over a minimal amount of mixed-metal MIL-100(Fe, Ni) MOF (0.001 $\mathrm{Fe}+\mathrm{Ni}$ metal $\mathrm{mmol}$ ) was performed. Under these extreme conditions, full conversions were achieved at long reactions times (see Figure S20), reaching a turnover number of $1 \times 10^{4}$. Importantly, a selectivity of $100 \%$ was maintained over the reaction. It should be commented, that although the Prins reaction has been studied with other MOFs and zeolites, the selectivity of nopol has been found to decrease by the presence of adventitious Brönsted acidity that causes the undesirable isomerization and other side reactions of norbornadiene. Also, although a combination of Amberlyst 15 and montmorillonite has been reported as an efficient heterogeneous catalyst, ${ }^{41}$ the present mixed-metal MIL-100(Fe, Ni) is more versatile, still allowing for furhter optimization.
The mixed-metal MIL-100(Fe, Ni) MOF containing $\mathrm{Ni}$ and the parent MIL-100(Fe) material have been studied as heterogeneous catalysts in a model acid-catalyzed reaction such is the Prins reaction. It was observed that the initial reaction rate increases gradually with the percentage of $\mathrm{Ni}^{\prime \prime}$ incorporated in the material. An improvement of the catalytic activity of more than one order of magnitude has been determined in case of the mixed-metal MIL-100(Fe, Ni) catalyst $3(5 \% \mathrm{Ni})$. This catalytic behaviour originated from the insertion of new $\mathrm{Ni}$ "Lewis acid sites, is maintained over successive cycles and correlates with $\mathrm{CO}$ adsorption measurements. The improved catalytic activity may have its origin at the distortion originated upon inclusion of $\mathrm{Ni}^{\prime \prime}$ ions in the framework, although local electronic modifications are also expected to play a role. A future in-depth study to get a more exhaustive understanding of this catalytic improvement is envisioned.

It is proposed that the use of this simple and direct synthetic approach may be applicable in the preparation of other mixedmetal MOFs suitable as heterogeneous catalysts. Considering the wide use of MOFs as heterogeneous catalysts, the present results showing the enhancement of the catalytic activity in heterometallic MIL-100 open the way to prepare new

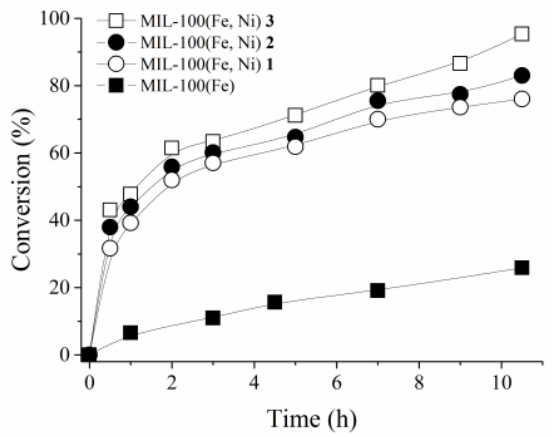

Figure 4. (a) Time conversion plot of $\beta$-pinene, using as catalyst either the mixed-metal MIL-100(Fe, Ni) 1-3 or homometallic MIL-100(Fe). (b) Hot filtration test and (c) reusability of mixed-metal material MIL$100(\mathrm{Fe}, \mathrm{Ni}) 3$ as catalyst for the $\beta$-pinene conversion. Reaction conditions: catalyst ( $15 \mathrm{mg}, 0.5 \mathrm{~mol} \%$ total metal based), $\beta$-pinene (1 $\mathrm{mmol}$ ) and paraformaldehyde $(1 \mathrm{mmol})$ in $\mathrm{MeCN}$ at $80^{\circ} \mathrm{C}$. Legend $(\mathrm{a})$ : MIL-100(Fe) (घ), MIL-100(Fe, Ni) 1 (o), $2(\bullet)$ and $\mathbf{3}(\square)$; Legend (b): 1st use $(\square)$, 2nd use $(\bullet)$, 3th use $(\square), 4$ th use $(0)$ and 5 th use $(\bullet)$. (inset) XRPD patterns of the fresh MIL-100(Fe, Ni) (black pattern) and after $2^{\text {nd }}$ and $5^{\text {th }}$ reuse (red and blue pattern, respectively).

\section{Conclusions}

Heterometallic Fe"l'/Ni" MOFs of the MIL-100 type material with different metal ratio have been synthesized, exhibiting the thermal, chemical and structural stability of the parent MIL$100(\mathrm{Fe}) \mathrm{MOF}$. The incorporation of $\mathrm{Ni}^{\prime \prime}$ centers in the heterometallic MIL-100(Fe"II', Ni") structure was based on analytical data, EXAFS and in-situ IR analysis, establishing both, the homogeneous distribution throughout the network and their accessible Lewis acid character.

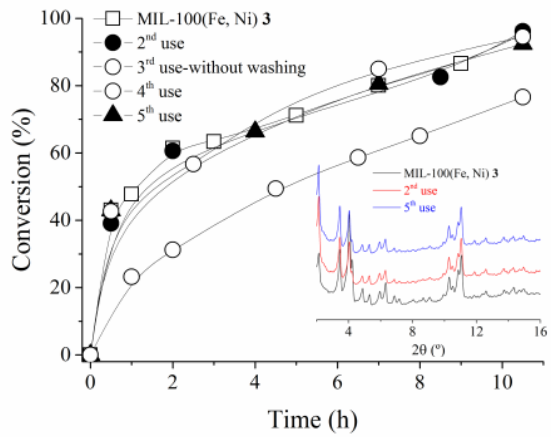

generations of even more active materials by introducing appropriate amounts of two or more metals at the nodes.

\section{Methods}

Materials. Iron powder, 1,3,5-benzenetricarboxylic acid (95\%) (trimesic acid), $\mathrm{HNO}_{3} \quad(65 \%)$ aqueous solution, paraformaldehyde, paraformaldehyde and $\beta$-pinene were purchased from Sigma-Aldrich. Solvents were used without further purification.

Synthesis of MIL-100(Fe) and mixed-metal MIL-100(Fe, Ni) MOFs (1-3). The homometallic MIL-100(Fe) MOF was obtained by directly mixing $1.680 \mathrm{~g}$ of trimesic acid $(8 \mathrm{mmol})$ and $0.670 \mathrm{~g}$ of Fe powder (12 mmol) in the case of MIL-100(Fe) in $90 \mathrm{~mL}$ of distilled $\mathrm{H}_{2} \mathrm{O}$, followed by the addition of a $65 \% \mathrm{HNO}_{3}$ aqueous solution $(0.424 \mathrm{~mL}, 6 \mathrm{mmol})$. The whole mixture was then heated to $85^{\circ} \mathrm{C}$ for 24 hours. After cooling to room temperature, the solution was filtrated and the obtained powder washed with water. The solid was then dispersed in $30 \mathrm{~mL}$ of $\mathrm{EtOH}$ and heated to $60{ }^{\circ} \mathrm{C}$ for 12 hours to remove the excess of trimesic acid. After cooling to room temperature, the resulting compound was recovered by filtration and washed with water. For the mixed-metal MIL-100(Fe/Ni) MOFs 1-3 different molar 
$\mathrm{M}^{\prime \prime \prime} / \mathrm{M}^{\prime \prime}$ mixtures were used: $1(11.88$ and $0.12 \mathrm{mmol}$ of $\mathrm{Fe}$ powder and $\mathrm{Ni}^{\prime \prime}$ acetate, respectively); 2 (11.4 and $0.6 \mathrm{mmol}$ ); 3 (10.8 and $1.2 \mathrm{mmol}$ ); The amount of $\mathrm{HNO}_{3}$ aqueous solution added was adjusted in all cases to 0.5 of the mmol of $\mathrm{Fe}^{0}$. MIL100 is formulated as: $\mathrm{Fe}_{3-\mathrm{x}} \mathrm{Ni}_{x} \mathrm{O}\left(\mathrm{C}_{6} \mathrm{H}_{3}\left(\mathrm{CO}_{2}\right)_{3}\right)_{2}(\mathrm{OH})_{x} \cdot\left(\mathrm{H}_{2} \mathrm{O}\right)_{3}$ $x \cdot($ solvent)

In-situ IR spectroscopic analysis. For in-situ IR analyses, samples were pressed into self-supported wafers $(4-10 \mathrm{mg} \mathrm{cm}-2)$ and treated in vacuum $(10-6 \mathrm{mbar})$ at $150{ }^{\circ} \mathrm{C}$ or $230{ }^{\circ} \mathrm{C}$ for $12 \mathrm{~h}$. NOFTIR spectra were re-corded by using a Nexus 8700 FTIR spectrophotometer using an IR cell that allowed us to perform in situ treatments at a controlled temperature and connected to a high vacuum system with a gas-dosing facility. Transmission IR spectra were recorded in the $500-5600 \mathrm{~cm}^{-1}$ range, at $4 \mathrm{~cm}^{-1}$ resolution. After activation, the wafers were cooled to $-125^{\circ} \mathrm{C}$ under dynamic vacuum followed by NO dosing at an increasing pressure (0.4-6 mbar). IR spectra were collected after each dosage. All IR spectra that correspond to the NO adsorption measurement were normalized to the weight of the wafer.

Thermogravimetric analysis. The thermal stability was evaluated by thermogravimetric analysis (TGA). TG measurements were carried out with a Mettler Toledo TGA/DSC 1 , STAR System apparatus under an $\mathrm{O}_{2}$ flow of $50 \mathrm{~mL} / \mathrm{min}$, at a heating rate of $3^{\circ} \mathrm{C} / \mathrm{min}$ to $500{ }^{\circ} \mathrm{C}$.

Powder X-Ray Diffraction analyses. Polycrystalline samples of MIL-100(FeNi) were lightly ground in an agate mortar and pestle and filled into $0.5 \mathrm{~mm}$ borosilicate capillaries prior to being mounted and aligned on a Empyrean PANalytical powder diffractometer, using Cu K $\alpha$ radiation $(\lambda=1.54056 \AA$ ). For each sample, three repeated measurements were collected at room temperature $\left(2 \theta=2-20^{\circ}\right)$ and merged in a single diffractogram. Adsorption measurements. Adsorption/desorption isotherms for $\mathrm{N}_{2}$ were measured at $77 \mathrm{~K}$ using a Tristar (Micromeritics). Samples were thoroughly dehydrated by heating at $150{ }^{\circ} \mathrm{C}$ for $5 \mathrm{~h}$ under vacuum. Adsorption/desorption isotherms for $\mathrm{CO}$ were measured at $303 \mathrm{~K}$ using a Quantachrome Autosorb Automated (Quantachrome Instruments). Samples were dehydrated by heating at $150{ }^{\circ} \mathrm{C}$ for $12 \mathrm{~h}$ under vacuum.

EXAFS experiments. The $\mathrm{Ni}$ and Fe $\mathrm{K}$ edges XAS measurements were performed at the ROCK beamline at the SOLEIL synchrotron radiation facility. ${ }^{42}$ Pellets of the powdered samples diluted with boron nitride were prepared with sample masses optimized in order to achieve an edge jump of about 1 for the Fe K edge and 0.1 to 0.2 at the $\mathrm{Ni} \mathrm{K}$ edge. Data were collected at Room Temperature in transmission mode using a Si(111) channel cut monochromator and ionization chambers filled with nitrogen. Harmonic rejection was ensured using two mirrors with $\mathrm{B}_{4} \mathrm{C}$ stripes aligned to $2.8 \mathrm{mrad}$ with respect to the incident beam. XAS analysis was carried out using the Athena software. ${ }^{43}$ Energy calibration was done at the tabulated values using the first derivative maximum of iron $\left(E_{0}=7112 \mathrm{eV}\right)$ and nickel $\left(E_{0}=8333 \mathrm{eV}\right)$ metallic foils located between the second and third ionization chambers and recorded simultaneously with the samples. After linear pre-edge and atomic background removal, XANES spectra were normalized in the middle of the first EXAFS oscillation. Fe K edge (resp. Ni) EXAFS spectra were $\mathrm{k}^{3}$-weighted before Fourier-transformation using a Kaiser-Bessel window in the $3.3-11$ (resp. 10) $\AA^{-1} \mathrm{k}$ range with $\mathrm{dk}=2$. Distances and Debye-Waller factors of the different contributions characterizing the local order of MIL-100(Fe) and mixed-metal MIL-100(Fe, Ni) MOFs (2-3) were determined by least-squares refinements using the Artemis interface to $\mathrm{IFeFFIT}^{42}$ whereas coordination numbers of those contributions were fixed at the values described in the MIL-100(Fe) crystallographic structure..$^{44}$ The amplitude reduction factors were obtained by fitting the EXAFS spectra of $\alpha-\mathrm{Fe}_{2} \mathrm{O}_{3}$ and $\mathrm{NiO}$ and their values $\left(\mathrm{S}_{0}{ }^{2}=0.68\right.$ (Fe $\mathrm{K}$ edge) and 1.23 ( $\mathrm{Ni} \mathrm{K}$ edge)) were kept fixed for the simulation of MIL-100(Fe) and mixed-metal MIL-100(Fe, Ni) MOFs (2-3) whereas the energy shift values which allows the match between theoretical and experimental k-scales were allowed to vary within the limits $7127.7 \pm 3.0 \mathrm{eV}$ and $8345.6 \pm$ $3.4 \mathrm{eV}$. The reliability of the fits was assessed by the minimization of the EXAFS reliability factor $R_{f}$ which measures the relative misfit with respect to the data and the reduced $\chi^{2} v$ quality factor, two metrics defined by the IXS standards and criteria committee. ${ }^{45}$

Catalytic experiments. The required amount of catalyst (typically $10 \mathrm{mg}$, except for productivity tests) was added to a round-bottom flask $(25 \mathrm{~mL})$. Catalyst activation was performed at $150 \stackrel{\circ}{ } \mathrm{C}$ under vacuum overnight. The reaction system was purged with molecular oxygen using a balloon, the substrate introduced $(20 \mathrm{mmol})$ and the system sonicated for $5 \mathrm{~min}$. Then, the reaction mixture was placed in a pre-heated bath at $80 \cong \mathrm{C}$ and magnetically stirred.

Reusability of MIL-100(Fe, Ni) was performed under the general reaction conditions. At the end of the reaction the catalyst was filtered through a Nylon membrane filter of $0.2 \mathrm{~mm}$. Then, the catalyst was washed by suspending the solid in methanol at 60 oC for $2 \mathrm{~h}$. This washing procedure was repeated two more times. Finally, the catalyst was dried at 100 ㅇ C overnight and used in subsequent catalytic cycles. Product analysis was conducted as follows: previously filtered reaction aliquots (100 $\mu \mathrm{L}$ ) were diluted in a MeCN solution $(500 \mu \mathrm{L})$ containing a known amount of nitrobenzene as the external standard. Then, the reaction aliquots were analyzed by gas chromatography using a flame ionization detector. Quantification was carried out by using calibration curves of authentic samples.

Leaching measurements. After reaction completion, the reaction mixture still hot was removed by filtration. Then, the organic phase was processed to extract the metal ions by stirring with a nitric aqueous solution $\left(\mathrm{HNO}_{3}, 3 \mathrm{M}\right)$ at $80{ }^{\circ} \mathrm{C}$ overnight. The metal content of the aqueous phase was analyzed by chemical analysis using an ICP-AES instrument.

Sheldon's hot filtration test. During this test, the catalyst was removed from the reaction by filtration while hot after $60 \mathrm{~min}$ of reaction, and the filtrate was monitored for continued activity.

\section{Conflicts of interest}

There are no conflicts to declare.

\section{Acknowledgements}


This work was supported by the European Commission under the Marie Sklodowska-Curie agreement H2020-MSCA-IF658224. Measurements at the ROCK synchrotron beamline of SOLEIL was supported by a public grant overseen by the French National Research Agency (ANR) as part of the "Investissements d'Avenir" program (reference: ANR10-EQPX45).

\section{Notes and references}

\footnotetext{
${ }^{1}$ See CrystEngComm 2017 themed issue : Metal-organic framework catalysis.

2 L. Zhu, X.-Q. Liu, H.-L. Jiang and L.-B. Sun, Chem. Rev., 2017, 117, 8129.

${ }^{3}$ H. García, S. Navalón, 2018, Ed. Wiley. ISBN: 978-3-527-80910-3.

${ }^{4}$ S. M. J. Rogge, A. Bavykina, J. Hajek, H. Garcia, A. I. Olivos-Suarez, A. Sepúlveda-Escribano, A. Vimont, G. Clet, P. Bazin, F. Kapteijn, M. Daturi, E. V. Ramos-Fernandez, F. X. Llabrés i Xamena, V. Van Speybroeck, J. Gascon, Chem. Soc. Rev., 2017, 46, 3134-3184.

${ }^{5}$ W. Tu, Y. Xu, S. Yin, and R. Xu, Adv. Mater. 2018, 30, 1707582.

${ }^{6}$ H. Depauw, I. Nevjestić, G. Wang, K. Leus, F. Callens, E. De Canck, K. De Buysser, H. Vrielinck, P. Van Der Voort, J. Mater. Chem. A, 2017, 5, 24580-24584.
}

${ }^{7}$ C. Huang, R. Liu, W. Yang, Y. Li, J. Huang, H. Zhu, Inorg. Chem. Front., 2018, 5, 1923-1932.

${ }^{8}$ A. Dhakshinamoorthy, A. M. Asiric and H. Garcia, Catal. Sci. Technol., 2016, 6, 5238.

${ }^{9}$ P. Silva, S. M. F. Vilela, J. P. C. Tomé, F. A. Almeida Paz, Chem. Soc. Rev., 2015,44, 6774-6803.

${ }^{10}$ Y. -B. Huang, J. Liang, X.-S. Wang and R. Cao, Chem. Soc. Rev., 2017, 46, 126.

${ }^{11}$ L. M. Aguirre-Díaz, F. Gándara, M. Iglesias, N. Snejko, E. GutiérrezPuebla M. A. Monge, J. Am. Chem. Soc., 2015, 137, 6132-6135.

12 L. Mitchell, P. Williamson, B. Ehrlichov, A. E. Anderson, V. R. Seymour, S. E. Ashbrook, N. Acerbi, L. M. Daniels, R. I. Walton, M. L. Clarke and P. A. Wright, Chem.- Eur. J., 2014, 20, 17185-17197.

${ }^{13}$ A. Schejn, A. Aboulaich, L. Balan, V. Falk, J. Lalevée, G. Medjahdi, L. Aranda, K. Mozet and R. Schneider, Catal. Sci. Technol., 2015, 5, 1829-1839.

14 J. Park, J.-R. Li, Y.-P. Chen, J. Yu, A. A. Yakovenko, Z. U. Wang, L.-B. Sun, P. B. Balbuena, H.-C. Zhou, Chem. Commun., 2012, 48, 99959997.

15 C. Castillo-Blas, V. A. de la Peña-O'Shea, I. Puente-Orench, J. Romero de Paz, R. Sáez-Puche, E. Gutiérrez-Puebla, F. Gándara, M. Monge, Sci. Adv. 2017,3:e1700773.

${ }^{16}$ L. M. Aguirre-Díaz, F. Gándara, M. Iglesias, N. Snejko, E. Gutiérrez-
Puebla, M. Monge, J. Am. Chem. Soc. 2015, 137, 6132-6135.

17 C. K. Brozek, M.Dincă, Chem. Soc. Rev. 2014, 43, 5456-5467.

18 S. M. Cohen Chem. Rev., 2012, 112, 970.

19 M. Mon, J. Ferrando-Soria, M. Verdaguer, C. Train, C. Paillard, B. Dkhil, C. Versace, R. Bruno, D. Armentano, E. Pardo, J. Am. Chem. Soc., 2017, 139,8098-8101.

20 P. Á. Szilágyi, P. Serra-Crespo, J. Dugulan, J. Gascon, H. Geerlingsad, B. Dama, CrystEngComm, 2013, 15, 10175-10178.

21 J. Castells-Gil, N. M. Padial, N. Almora-Barrios, J. Albero, A. R. RuizSalvador, J. González-Platas, H. García, C. Martí-Gastaldo Angew. Chem. Int. Ed. 2018, 57, 8453.

${ }^{22}$ C. Serre, F. Millange, S. Surblé and G. Férey, Angew. Chem., Int. Ed., 2004, 43, 6296.

${ }^{23}$ S. Surblé, C. Serre, C. Mellot-Draznieks, F. Millange, G. Férey, Chem. Commun., 2006, 0, 284-286.

24 V. Guillerm, S. Gross, C. Serre, T. Devic, M. Bauer, G. Férey, Chem. Commun., 2010, 46, 767.

25 S. Wongsakulphasatch, F. Nouar, J. Rodriguez, L. Scott, C. Le Guillouzer, T. Devic, P. Horcajada, J.-M. Grenèche, P.L. Llewellyn, A. Vimont, G. Clet, M. Daturi, C. Serre, Chem. Commun., 2015, 51, 10194.

${ }^{26}$ D. Feng, K. Wang, Z. Wei, Y.-P. Chen, C. M. Simon, R. K. Arvapally, R. L. Martin, M. Bosch, T.-F. Liu, S. Fordham, D. Yuan, M. A. Omary, M. Haranczyk, B. Smit and H.-C. Zhou, Nat. Commun. 2014, 5:5723.

27 X.-L. Wang, L.-Z. Dong, M. Qiao, Y.-J. Tang, J. Liu, Y. Li, S.-L. Li, J.-X. Su, Y.-Q. Lan, Angew.Chem.Int. Ed. 2018, 57, 9660.

${ }^{28}$ H. Vrubel, T. Hasegawa, E. Oliveira, F. S. Nunes, Inorg. Chem. Commun. 2006, 9, 208.

${ }^{29}$ A. Schoedel and M. J. Zaworotko, Chem. Sci., 2014, 5, 1269.

${ }^{30}$ W. Zhang, Y. Shi, C. Li, Q. Zhao, X. Li, Catal. Lett., 2016, 146, 1956.

${ }^{31}$ F. Nouar, T. Devic, H. Chevreau, N. Guillou, E. Gibson, G. Clet, M. Daturi, A. Vimont, J.M. Grenèche, M. I. Breeze, R. I. Walton, P. L. Llewellyne and C. Serre, Chem. Commun., 2012, 48, 10237.

32 (a) M. Opanasenko, A. Dhakshinamoorthy, Y. K. Hwang, J.-S. Chang, H. Garcia and J. Čejka, ChemSusChem, 2013, 6, 865. (b) G. GómezPozuelo, C. Palomino Cabello, M. Opanasenko, M. Horáček, J. Čejka, 
Chang, G. F.rey, and M. Daturi, Chemistry 2012, 18, 11959.

39 J. F. Eubank, P. S. Wheatley, G. Lebars, A. C. McKinlay, H. Leclerc, P. Horcajada, M. Daturi, A. Vimont, R. E. Morris, C. Serre, APL Materials 2, 2014, 124112.

${ }^{40}$ Common Fragrance and Flavor Materials, Preparation, Properties, and Uses (Eds.: H. Surburg, J. Panten), Wiley-VCH, Weinheim, 2006, 67.

${ }^{41}$ J. S. Yadav, B.V. Subba Reddy, G. Mahesh Kumar, Ch.V.S.R Murthy, Tetrahedron Letters, 2001, 42, 89.

${ }^{42}$ V. Briois, C. La Fontaine, S. Belin, L. Barthe, T. Moreno, V. Pinty, A. Carcy, R. Girardot, E. Fonda, Journal of Physics Conferences Series, 2016, 712: art. $n^{\circ} 012149$.

ChemPlusChem 2017, 82,152.

33 Jing Shi, Shengtao Hei, Huanhuan Liu, Yanghe Fu, Fumin Zhang, Yijun Zhong, and Weidong Zhu, Journal of Chemistry, 2013, Article ID 792827.

${ }^{34}$ L. Peng, M. Asgari, P. Mieville, P. Schouwink, S. Bulut, Daniel T. Sun, ${ }^{43}$ B. Ravel, M. Newville, J. Synchrotron Radiat., 2005, 12, 537.

44 P. Horcajada, S. Surblé, C. Serre, D.-Y. Hong, Y.-K. Seo, J.-S. Chang, J.-M. Grenèche, I. Margiolakid G. Férey, Chem. Commun., 2007, 2820.

45 http://ixs.iit.edu/subcommittee_reports/sc/err-rep.pdf Zhongrui Zhou, Philip Pattison, Wouter van Beek, and Wendy L. Queen, ACS Appl. Mater. Interfaces, 2017, 9, 23957.

35 (a) J. F. Eubank, P. S. Wheatley, G. Lebars, A. C. Mckinlay, H. Leclerc, P. Horcajada, M. Daturi, A. Vimont, R. E. Morris and C. Serre, APL Mater., 2014, 2, 124112. (b) C. Serre, P. Horcajada, G. FeÅLrey, A. Vimont, M. Daturi, P.L. Llewellyn, Y.K. Hwang, J.-W. Yoon, J. S. Chang, 106660/FR PCT/FR2010/000402 (28/05/2010).

${ }^{36}$ K. I. Hadjiivanov, Catal. Rev.: Sci. Eng., 2000, 42, 71.

37 J. W. Yoon, Y.-K. Seo, Y. K. Hwang, J.-S. Chang, H. Leclerc, S. Wuttke, P. Bazin, A. Vimont, M. Daturi, E. Bloch, P. L. Llewellyn, C. Serre, P. Horcajada, J.-M. Grenche, A. E. Rodrigues, G. Férey, Angew. Chem. Int. Ed. 2010, 49, 5949

${ }^{38}$ S. Wuttke, P. Bazin, A. Vimont, C. Serre, Y. Seo, Y. K. Hwang, J. S. 